Review

\title{
Non-clinical assessment of cell therapy products: the perspective from five Asian countries/regions based on regulatory guidelines and the underpinning rationales
} \author{
James Ooi ${ }^{15}$, Yoshie Tsurumaki ${ }^{1}$ \\ ${ }^{1}$ Translational Medicine, Novartis Institutes for BioMedical Research, Novartis Pharma K.K., Tokyo, Japan \\ ${ }^{2}$ Strategic Center for Regenerative Medicine, Inha University College of Medicine, Incheon, South Korea \\ ${ }^{3}$ Stempeutics Research, Bangalore, India \\ ${ }^{4}$ Steminent Biotherapeutics, Inc, Taipei, Taiwan \\ ${ }^{5}$ Help Therapeutics Co, Ltd, Nanjing, China \\ ${ }^{6}$ Daiichi Sankyo Co, Ltd, Tokyo, Japan \\ ${ }^{7}$ Takeda Pharmaceutical Company Limited, Fujisawa, Japan \\ ${ }^{8}$ Sumitomo Dainippon Pharma Co, Ltd, Kobe, Japan \\ ${ }^{9}$ Astellas Pharma Inc, Tsukuba, Japan \\ ${ }^{10}$ Kangstem Biotech, Seoul, South Korea \\ ${ }^{11}$ Development Center for Biotechnology, Taipei, Taiwan \\ ${ }^{12}$ SCM Lifescience Inc, Incheon, South Korea \\ ${ }^{13}$ Xcell Therapeutics, Seoul, South Korea \\ ${ }^{14}$ Beijing Baylx Biotech Co, Ltd, Beijing, China \\ ${ }^{15}$ Novartis Institutes for BioMedical Research, Shanghai, China
}

Tomohiro Yoneda ${ }^{1, *}$, Byung H. Choi ${ }^{2}$, Pawan K. Gupta ${ }^{3}$, Chih-Yuan Ho ${ }^{4}$, Yat P. Tsui ${ }^{5}$, Ling-Mei Wang ${ }^{4}$, Yukari Fujiwara ${ }^{1}$, Hiroshi Karasawa ${ }^{6}$, Yuu Moriya ${ }^{7}$, Kiyoko Bando ${ }^{8}$, Yoshiteru Kamiyama ${ }^{9}$, Masayuki Kanki ${ }^{9}$, Kou Omura ${ }^{9}$, Takeshi Watanabe ${ }^{7}$, Yohan Bae ${ }^{10}$, Feng-Cheng Chou ${ }^{11}$, Dong-sik Ham ${ }^{12}$, Joo Y. Lee ${ }^{13}$, Guangyang Liu ${ }^{14}$, Yongjun Liu ${ }^{14}$,

\section{A R T I C L E I N F O}

\section{Article History:}

Received 14 January 2021

Accepted 20 April 2021

\section{Key Words:}

APACRM

Asia

cell therapy product

non-clinical assessment

regulatory guideline

\begin{abstract}
A B S T R A C T
Background aims: Cell-based regenerative medicine is an innovative field that can potentially alter the overall survival and quality of life of patients with devastating diseases. Several cell therapy products (CTPs) have been approved within the last two decades, and more are under development. The establishment of an effective developmental strategy in accordance with the regulatory bodies of each country/region is crucial for fast delivery of each respective CTP. In particular, facilitating investigational new drug (IND) approval is important for accelerating the transition from non-clinical to clinical research/trial phases.

Methods: Here the authors compared the non-clinical prerequisites for initiating clinical studies in five Asian countries/regions (India, China, Korea, Taiwan and Japan) from an industry viewpoint. The authors first identified the differences and tried to clarify the perspectives/considerations underpinning the different requirements. Results: The authors' findings revealed that differences in regulations and development experiences, especially with CTPs, have led to clear differences in the non-clinical study package and its corresponding study design. Conclusions: By sharing experiences of the research and development of CTPs among Asian countries/regions and including not only industry but also regulatory authorities, we will be able to expedite cross-border IND approval and eventually contribute to the early delivery of innovative CTPs to many Asian patients.

(c) 2021 International Society for Cell \& Gene Therapy. Published by Elsevier Inc. This is an open access article under the CC BY-NC-ND license (http://creativecommons.org/licenses/by-nc-nd/4.0/)
\end{abstract}

\footnotetext{
* Correspondence: Tomohiro Yoneda, MSc, Translational Medicine, Novartis Institutes for BioMedical Research, Novartis Pharma K.K., Toranomon Hills Mori Tower, 123-1, Toranomon, Minato-ku, Tokyo, Japan.

E-mail address: tomohiro.yoneda@novartis.com (T. Yoneda).
}

\section{Introduction}

Regenerative medicine is a fast-growing field of the past decade that leverages technical advancements to cure patients with devastating diseases [1-3]. Cell therapy products (CTPs) are one of the 


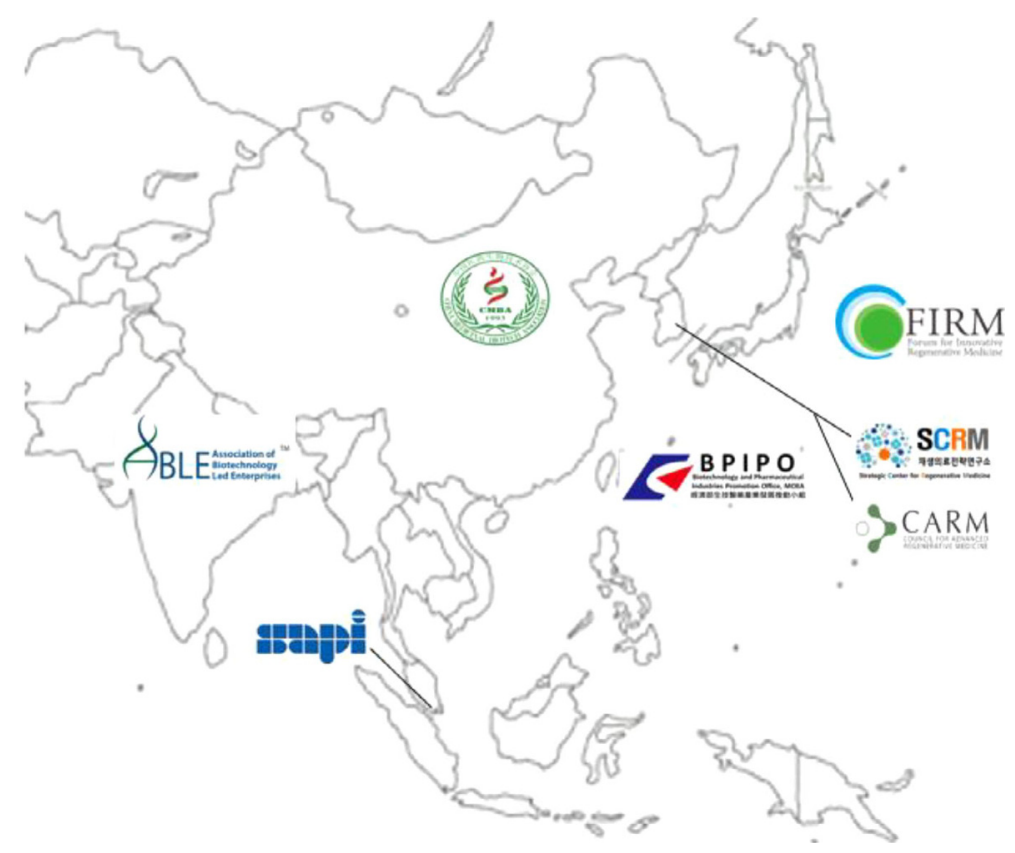

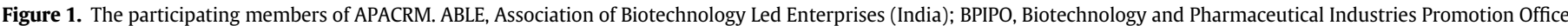

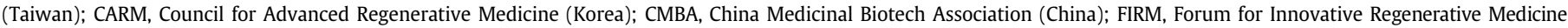
(Japan); SAPI, Singapore Association of Pharmaceuticals Industries (Singapore); SCRM, Strategic Center for Regenerative Medicine (Korea). (Color version of figure is available online).

main pillars of this field, and some CTPs have already been approved worldwide through multi-national clinical trials or domestically through local clinical trials [4]. Currently, there are a growing number of clinical trials for CTPs, and as such, regulatory guidelines for specific countries/regions have been issued to support the development path of CTPs [5,6]. However, there has been very little effort in unifying the regulation of regenerative medicine, including CTPs, among different countries/regions. Therefore, it is common that a CTP development strategy is acceptable in some countries/regions but not in others because of differences in regulatory requirements. In such circumstances, the establishment of separate development strategies for CTPs in accordance with different local regulations is indispensable for delivering CTPs to patients in different countries/regions.

The Asia Partnership Conference of Regenerative Medicine (APACRM) was established in 2018 with the intention of contributing to the regulatory harmonization of regenerative medicine products across Asia. The local leading industry associations for regenerative medicine are members of this conference (Figure 1). The mission of APACRM is to support the development and delivery of quality regenerative medicine products to all Asian patients. Therefore, it is important to promote harmonization and optimization of regulations regarding regenerative medicine products throughout Asia.

Data from research and development of CTPs must undergo vigorous validation to ensure quality, safety and efficacy. A consultation with the appropriate regulatory agency to proceed with the next phase of development is an indispensable task for sponsors developing CTPs. In particular, the efficient acceptance of investigational new drug (IND) applications is important for accelerating the transition from non-clinical to clinical research/trial phases.

APACRM working group 1 (WG1) was launched in June 2019 and consists of industrial professionals from India, China, Korea, Taiwan and Japan with expertise in non-clinical assessment of regenerative medicine products, including CTPs. Given the recent advancements in CTPs, the main objective of APACRM WG1 is to investigate differences in non-clinical requirements for CTP registration across Asia and identify the considerations and hurdles from the viewpoint of regulatory science. Here the authors summarize and compare the local perspectives, considerations and guidelines for non-clinical assessment of human CTPs entering clinical trials from an industry viewpoint. For sponsors developing CTPs in Asia, this information will serve as a practical reference for preparing a non-clinical data package that is in line with the different local regulations in Asia.

\section{Two Virtual CTP Models}

When considering the inherent complexity and diverse nature of CTPs, a CTP development strategy is based on the specific product profile on a case-by-case basis. Here the authors employed a case study approach using two virtual CTPs as model products by optimizing and omitting case settings for each model. These virtual models can help us identify the basic considerations of the different regulatory bodies without the constraints of a real CTP and gain further insights into the non-clinical assessment of CTPs apart from the specific CTP profile.

First, among several sources of cells for human CTPs, the authors selected two contrasting cell types: mesenchymal stromal cells (MSCs) and induced pluripotent stem cells (iPSCs). MSCs have a long history as a cell source for CTPs, and several products have already been marketed in Asia [7,8]. Therefore, both regulatory authorities and industries have abundant experience and knowledge regarding this cell type. By contrast, the overall development of iPSC-derived CTPs is still premature [9-11]. The pluripotency and infinite selfrenewal capacity of iPSCs affect the non-clinical strategy of such CTPs, particularly in terms of safety assessment [12].

The profiles of the two virtual CTPs were created based on their typical characteristics and to highlight their contrasting differences to fully understand the different perspectives of each country/region (Table 1). As a general consideration of the products, target patients were adults and target indications were set as incurable diseases or states with no currently available therapies. The model cases have no approved CTPs and thus have no practical reference products. To simplify these virtual CTPs, no genetic manipulation was stipulated. The ectopic expression of specific genes in the initial creation of iPSCs and the in-process impurity are not within the scope of this virtual exercise.

In the case of MSC-derived CTPs, allogeneic and adipose tissuederived cells are normally employed for clinical use [7]. Rheumatoid 
Table 1

Virtual CTP profiles.

\begin{tabular}{lll}
\hline Product profile & Virtual model 1: MSC-derived product & Virtual model 2: iPSC-derived product \\
\hline Donor type & Allogeneic & Autologous \\
Development phase & First-in-human & First-in-human \\
Cell source & Adipose tissue-derived MSCs & Human blood cell-derived iPSCs \\
Proliferation activity & Low & High \\
Mechanism of action & Immunosuppressive effects mediated by cellular secretions & Engraftment of progenitor cells supplying functional cells \\
Indication & Rheumatoid arthritis (adults) & Hematologic disease (adults) \\
Clinical administration route & Local (intra-articular) & Systemic (intravenous) \\
Clinical administration cell number & Low: $1 \times 10^{6}$ cells/body & High: $1 \times 10^{9}$ cells/body \\
Clinical administration frequency & Monthly, six doses & Single dose \\
Animal pathogenic model & Yes & No \\
Genetic manipulation & No & No \\
\hline
\end{tabular}

arthritis was selected as an indication because there are many patients who are resistant/refractory to biological drugs and a validated animal model is available $[13,14]$. The mechanism of action (MOA) of the MSC-derived virtual CTP was defined as immunomodulatory effects via paracrine factors based on previous reports $[15,16]$. The dosing regimen in the clinical study was determined based on the chronic nature of the disease. For the iPSC-derived CTP, autologous differentiated cells were selected to avoid the complexity arising from immune rejection of allogeneic cells. Hypothetical hematologic disease caused by a lack of specific functional cells in adults was set as a target indication to emphasize the effectiveness of supplementing progenitor cells generated from iPSCs. Systemic administration of a high-dose regimen is very challenging and has not been attempted in clinical practice. However, using this virtual exercise to investigate the conditions under which the clinical cell dose exceeds the maximum feasible dose (MFD) in animals is of interest to the field.

\section{Questionnaire on the Non-Clinical Assessment of Virtual CTPs}

After establishing the virtual CTPs, the authors created a questionnaire to investigate the local non-clinical assessments needed for submitting an IND application. The questionnaire asked the participating respondents to describe the necessary in vitro and in vivo non-clinical studies to assess pharmacology, cellular kinetic (CK) and biodistribution (BD) studies and safety of the CTPs (Figure 2). The questionnaire was filled out based on the local regulatory guidelines and experience with CTP development of five Asian countries/regions (India, China, Korea, Taiwan and Japan). The authors then compared the questionnaires to identify the rationales behind the different answers.

\section{The Regulatory Landscape}

Before presenting the questionnaire answers, the authors will briefly summarize the Asian regulatory landscape of CTP development in the following sections. Although there are no available international regulatory documents for CTPs in Asia, there are domestic regulatory guidelines regarding the non-clinical requirements of CTP development (Table 2). The optimal strategy for nonclinical assessment is known to depend on the unique properties and risk profiles of each CTP and intended recipient.

\section{Regulation in India}

There are no separate guidelines for conducting non-clinical studies of CTPs in India. Instead, the guidelines are complementary to the existing "New Drugs and Clinical Trial Rules 2019" issued by the Ministry of Health and Family Welfare and the "National Guidelines of Stem Cell Research 2017" issued by the Indian Council of Medical Research and Department of Biotechnology [17,18].

The minimum non-clinical studies to be completed before conducting clinical trials with CTPs will be discussed here. All non-clinical safety and efficacy studies for assessing CTPs are similar to those of traditional modalities, such as biologics (i.e., single- and repeateddose toxicity studies, genotoxicity, immunogenicity and prenatal development toxicity studies), in addition to tumorigenicity studies. Safety studies include toxicity studies (acute and subchronic singleand repeated-dose toxicity studies) in rodents and non-rodents. Dosages can be a single dose of up to 20 times the therapeutic dose (TD) planned for humans for acute toxicity studies or repeated doses if the CTP is planned for use as multiple doses. The studies are to be performed using the same route of administration (ROA) as that intended for humans. In addition, unless the intended ROA is only intravenous, at least one more ROA should be tested to ensure systemic absorption of the drug. Animals should be observed for 14 days after CTP administration, and the minimum lethal dose and maximum tolerated dose (MTD) should be established. Based on the data of the acute toxicity studies, dosing for the subchronic studies can be determined, which can be low $(1 \times \mathrm{TD})$, mid $(5 \times \mathrm{TD})$ or high $(10$ or
Q. Are non-clinical evaluations (pharmacology, CKBD, and safety) needed prior to starting clinical trials in your country/region?

Q. Please describe the type of studies (e.g., in vitro, in vivo, ex vivo) needed.

Q. Should the studies be conducted according to GLP?

Q. How many animal species are required per study item?
Q. Which animal species and models are required for each study type?

Q. What is the dosage to be tested and how many dosage groups should be set per study?

Q. Please describe the administration route, dosing regimen, and items to be measured in nonclinical studies.

Q. How long must the observational period be per study?

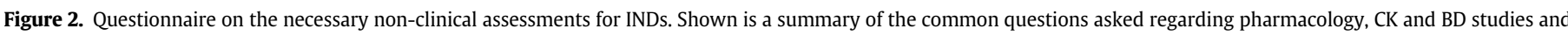
safety tests for CTPs. Refer to Table 3 and supplementary Table 1 for more details regarding the questions and answers. (Color version of figure is available online). 
Table 2

List of local regulatory guidelines regarding CTPs.

\begin{tabular}{|c|c|c|}
\hline Respondent & Guideline & Reference \\
\hline \multirow[t]{3}{*}{ India } & 1. Draft Guidelines of Stem Cells and Cell-Based Product (2013, CDSCO) & [49] \\
\hline & 2. National Guidelines of Stem Cell Research (2017, ICMR) & [17] \\
\hline & 3. New Drug and Clinical Trial Rules (2019, CDSCO) & [18] \\
\hline \multirow[t]{3}{*}{ China } & 1. Guidance on CMC and Preclinical Study of Stem Cell (2015, NMPA) & [19] \\
\hline & 2. Guidance on Cell Therapy Products Study and Evaluation (draft) (2017, CDE) & {$[20]$} \\
\hline & 3. Draft Technical Guidelines for Clinical Trials of Human-Derived Stem Cells and Cell Therapy Products (2020, CDE) & {$[50]$} \\
\hline \multirow[t]{6}{*}{ Korea } & 1. Guideline in Quality, Non-Clinical and Clinical Assessment of Stem Cell Therapy Product (2014, MFDS) & {$[24]$} \\
\hline & 2. Considerations in Immunotoxicity Assessment of Allogenic Stem Cell Therapy Product (2014, MFDS) & [25] \\
\hline & 3. Considerations in Tumorigenicity Assessment of Stem Cell Therapy Product (2014, MFDS) & {$[28]$} \\
\hline & 4. Considerations in Biodistribution Assessment of Stem Cell Therapy Product (2014, MFDS) & [29] \\
\hline & 5. Guidance on Non-Clinical Assessment of Gene Therapies (2017, MFDS) & [23] \\
\hline & 6. Advanced Regenerative Medicine and Advanced Biopharmaceuticals Safety and Support Act (2020, MFDS) & [22] \\
\hline Taiwan & 1. Guidance on Investigational Cell Therapy Products (2020, TFDA) & [30] \\
\hline \multirow[t]{9}{*}{ Japan } & $\begin{array}{l}\text { 2. Guidelines on Ensuring the Quality and Safety of Pharmaceuticals and Medical Devices Derived from the Processing of Autologous Human Cell/ } \\
\text { Tissue (2008, MHLW) }\end{array}$ & [31] \\
\hline & $\begin{array}{l}\text { 3. Guidelines on Ensuring the Quality and Safety of Pharmaceuticals and Medical Devices Derived from the Processing of Allogeneic Human Cell/ } \\
\text { Tissue (2008, MHLW) }\end{array}$ & [32] \\
\hline & $\begin{array}{l}\text { 4. Guidelines on Ensuring the Quality and Safety of Pharmaceuticals and Medical Devices Derived from the Processing of Autologous Human } \\
\text { Somatic Stem Cells (2012, MHLW) }\end{array}$ & {$[33,38]$} \\
\hline & $\begin{array}{l}\text { 5. Guidelines on Ensuring the Quality and Safety of Pharmaceuticals and Medical Devices Derived from the Processing of Allogeneic Human } \\
\text { Somatic Stem Cells (2012, MHLW) }\end{array}$ & {$[34,39]$} \\
\hline & $\begin{array}{l}\text { 6. Guidelines on Ensuring the Quality and Safety of Pharmaceuticals and Medical Devices Derived from the Processing of Autologous Human } \\
\text { Induced Pluripotent Stem (-Like) Cells (2012, MHLW) }\end{array}$ & {$[35,40]$} \\
\hline & $\begin{array}{l}\text { 7. Guidelines on Ensuring the Quality and Safety of Pharmaceuticals and Medical Devices Derived from the Processing of Allogeneic Human } \\
\text { Induced Pluripotent Stem (-Like) Cells (2012, MHLW) }\end{array}$ & {$[36,41]$} \\
\hline & $\begin{array}{l}\text { 8. Guidelines on Ensuring the Quality and Safety of Pharmaceuticals and Medical Devices Derived from the Processing of Human Embryonic Stem } \\
\text { Cells (2012, MHLW) }\end{array}$ & {$[37,42]$} \\
\hline & $\begin{array}{l}\text { 9. Technical Guidance on the implementation of the Quality, Non-Clinical and Clinical Study of Regenerative Medicine (Products Derived From } \\
\text { the Processing of Human Cells) (2016, MHLW) }\end{array}$ & [43] \\
\hline & $\begin{array}{l}\text { 10. Guidelines on the Detection of Undifferentiated Pluripotent Stem Cells and Transformed Cells, Tumorigenicity Tests and Genetic Stability } \\
\text { Evaluation on Human Cell Processed Products (2019, MHLW) }\end{array}$ & [44] \\
\hline
\end{tabular}

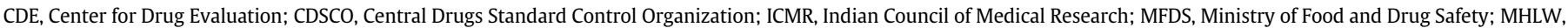
Ministry of Health, Labour and Welfare; NMPA, National Medical Products Administration; TFDA, Taiwan Food and Drug Administration.

$20 \times$ TD) doses. For tumorigenicity studies, doses to be tested should be similar to those of the toxicity studies and should include a positive control.

For testing non-clinical efficacy in animal models, the selected models should match the human disease and disease phenotype. The endpoints should match the clinical outcomes and demonstrate a mechanism of the treatment effect. Large animal models, such as non-human primates (NHPs), may be used when necessary. Further CK and BD studies of stem cells should be conducted using the intended ROA. Studies such as prenatal development toxicity studies may be required depending on the nature of the product and the proposed indication. Stem cells used for non-clinical studies should be well characterized, similar to those to be used in clinical trials, and manufactured in a Central Drugs Standard Control Organizationapproved manufacturing facility.

\section{Regulation in China}

In China, the regulatory guidelines are covered by two documents: "Guidance for the Research and Evaluation of Cell Therapy Products (Trial Implementation)" issued by the Center for Drug Evaluation and National Medical Products Administration in 2017 and "Guidance for the Quality Control and Preclinical Research of Stem Cell-Based Medicinal Products (Trial Implementation)" issued in $2015[19,20]$. The latter involves specific guidelines for stem cellbased medicinal products, whereas the former is all-encompassing, covering test article requirements and analysis, pharmacology, nonclinical and clinical studies and risk mitigation strategies. As most guidelines in the 2015 document are covered in the 2017 document, the authors will mainly focus on the 2017 document while highlighting guidelines that are unique in the other. The non-clinical section of the 2017 document covers the test articles (i.e., requirements, analysis and mode of administration), pharmacokinetics (PKs)/ pharmacodynamics (PDs) (i.e., implanted cell distribution, migration, homing and differentiation) and safety evaluation (i.e., safety pharmacology, single and repeated dose, immunogenicity, tumorigenicity, genotoxicity and reproductive toxicity).

Regarding the test articles, the guidelines stress the importance of using the same CTPs intended for clinical trials. The production process and quality control of the test articles used for non-clinical studies should be consistent with those intended for clinical trials, with explanations and predictions of potential clinical responses for any discrepancies. Cells of animal origin can be used as substitutes, but the procurement/production procedures and quality must be as similar as possible. In case the test article requires further processing before administration, quality control should be conducted.

The guidelines suggest aspects to consider when selecting appropriate animal species in non-clinical studies. For example, the animal species of choice should be anatomically similar or comparable to humans. Immunosuppressed or deficient animals and animal models of disease can be used with justification. The guidelines recognize the lack of scientific consensus in animal model selection for tumorigenicity studies. As for test article administration, the guidelines recommend using a similar administration method as the intended clinical application.

The guidelines further recommend the use of both in vitro and in vivo platforms for PD studies. In addition, the MOA, ROA and disease length should be considered. For PK and repeated-dose toxicity studies, animals of both sexes should be considered. Studies should focus on in vivo proliferation, distribution, migration and differentiation of target cells; the expression/secretion of biomolecules; and interactions with host tissues. The distribution and duration of CTPs are key factors affecting efficacy and safety. Thus, the guidelines stipulate that the length of single-dose toxicity studies should sufficiently cover the survival time of the implanted cells and their effects. The immunogenicity of CTPs and their secreted factors also need to be assessed. Moreover, 
the tumorigenicity risk of CTPs may vary depending on cell type, differentiation status, culture method used during manufacturing, gene modification, etc. It is thus recommended to select a suitable animal model, a suitable number of cells, a reasonable implantation route and a sufficiently long observation period to effectively evaluate CTP tumorigenicity. Given that effective evaluation of tumorigenicity requires long-term survival of the CTP in vivo, immunodeficient animals can be used for tumorigenicity studies.

Other toxicity studies and safety considerations recommended by the guidelines include, but are not limited to, (i) assessment of local tolerance of the CTPs and their secreted products, (ii) assessment of reproductive toxicity and (iii) assessment of genotoxicity. Genotoxicity should be carefully evaluated if the CTPs or their secretions directly or indirectly interact with the DNA of the recipient. For genetically modified CTPs, the risk of oncogenic activity caused by insertion mutations or residual gene-modifying vectors capable of replication must be evaluated.

The guidelines recognize the diversity of CTPs and recommend that non-clinical studies be designed specifically for each product based on the pharmacological and PK attributes as well as clinical application considerations. The document further refers to ICH S6 (R1), "Preclinical Safety Evaluation of Biotechnology-Derived Pharmaceuticals," as supplemental guidance [21].

\section{Regulation in Korea}

Korea recently enforced the "Advanced Regenerative Medicine and Advanced Biopharmaceuticals Safety and Support Act" in August 2020 [22]. The act regulates the overall processes and requirements for the approval and execution of clinical research/trials of cell and gene therapies. However, non-clinical studies of these advanced therapies, including non-clinical data on general toxicology, immunogenicity, tumorigenicity, efficacy, BD and pharmacology, are still regulated by the "Regulation on Review and Authorization of Biological Products" and "Enforcement Rule on Safety of Pharmaceuticals" under the Pharmaceutical Affairs Act.

Toxicological data must be submitted for the examination of safety, validity, standards and testing methods in accordance with the "Enforcement Rule of Safety of Pharmaceuticals." Non-clinical toxicity testing should be conducted to predict toxic reactions in humans, determine the range of safe doses in clinical trials and identify target organs and monitor factors that can become toxic in patients. The toxicological data should also be tested according to good laboratory practice (GLP).

The Ministry of Food and Drug Safety, the regulatory authority in Korea, has published additional guidelines on gene therapy and stem cell therapy under the Pharmaceutical Affairs Act. The "Guideline on Non-Clinical Assessment of Gene Therapy," published in December 2017, deals with basic principles and considerations regarding toxicology as well as pharmacology studies of in vivo and ex vivo gene therapies according to the different vector constructs [23]. The guidelines emphasize that an animal model and ROA with reasonable clinical relevance should be used to determine MOA, toxicology and therapeutic efficacy of the gene therapy. They also recommend using more than one animal model or a humanized animal model if available and to consider the in vivo behavior as well as the effects of all vector components in the study design and assays. Particular emphasis should be placed on genotoxicity, reproductive/developmental toxicity and tumorigenicity for toxicology studies as well as absorption, distribution, metabolism and excretion; BD; and virus shedding for pharmacology studies.

There are four additional guidelines for stem cell therapy. The "Guideline in Quality, Non-Clinical and Clinical Assessment of Stem Cell Therapy Product," published in December 2014, describes the overall requirements for non-clinical studies of stem cell therapies in consideration of the uncontrolled and unlimited proliferation ability of undifferentiated stem cells in vivo [24]. The basic principles for the non-clinical assessment of stem cell products are similar as those for gene therapy mentioned earlier, with additional considerations, such as transplant-host interactions in a specific niche, differentiation, allogeneic immunogenicity, persistence and tumorigenicity in vivo.

The Ministry of Food and Drug Safety provides guidelines for designing an immunotoxicity study of stem cell therapy in "Considerations in Immunotoxicity Assessment of Allogenic Stem Cell Therapy Product," published in September 2014 [25]. The guidelines are concerned with host immune responses against allogeneic stem cells in general. They also highlight the potential immunotoxicity of MSCs in association with unintended excessive immunosuppression by overdosing as well as immune-boosting effects resulting from changes in HLA expression at the inflammation area or sensitization via repeated administration [26,27].

"Considerations in Tumorigenicity Assessment of Stem Cell Therapy Product," published in September 2014, should be referred to when designing a tumorigenicity study of a stem cell therapy [28]. These guidelines recommend performing a tumorigenicity study in vivo for stem cells or any cell types/products with a potential risk of tumor formation while considering cell types and their differentiation profile, gene expression patterns, genomic stability, target tissues and patient groups. Immunocompromised or deficient animals are recommended, whereas humanized rodent models, disease models or the use of immunosuppressants is also accepted alone or in combination if available or unavoidable. ROA, dose and frequency should follow those of clinical settings. For cell-based therapies, the MFD should be included. When a suitable protocol is unavailable, the use of large animals or alternative ROA can be considered with scientific evidence and validation data. Appropriate negative and positive control groups should be included in the study design. Tumor cells or undifferentiated stem cells relevant to the target therapy are recommended as positive controls, and the acceptance criteria should be provided. The observation period should depend on the stem cell characteristics, and a sufficiently long follow-up period (e.g., $\geq 6$ months) is recommended.

For testing non-clinical efficacy, PDs and PKs of stem cell therapy, "Considerations in Biodistribution Assessment of Stem Cell Therapy Product," published in September 2014, can be referred to in addition to the regulations and rules mentioned earlier [29]. Both in vitro and in vivonon-clinical efficacy studies are recommended, and the data should provide evidence to confirm (i) the range of pharmacological activity by establishing an optimal dose and minimal effective dose, (ii) the optimal drug ROA for clinical studies and (iii) the dosing schedule for early-phase clinical studies. Although standalone safety pharmacology studies are not required for most advanced biopharmaceuticals, they can be conducted in accordance with the pharmacology standard for pharmaceuticals when needed to determine MOA. Specific concerns regarding the study design include the following: (i) animal disease models should be used to assess the BD of stem cells if available (otherwise, immunocompromised, immunodeficient or humanized rodents can be used); (ii) a sufficient number of experimental animals should be used to assess the sensitivity of testing materials, testing methods and variation of animals; (iii) ROA should reflect clinical ROA, but when technically unavailable, other ROAs are acceptable with a scientific rationale; (iv) dosage and frequency should reflect the clinical trial design; ( $v$ ) the observation period should last at least until the product is not detected in the assay; and (vi) when determining the observation period, the engraftment, growth and differentiation of cells should be considered, as they can vary depending on stem cell type.

\section{Regulation in Taiwan}

The Taiwan Food and Drug Administration issued the newly amended "Guidance on Investigational Cell Therapy Products" in 
May 2020, which includes the latest guidelines for non-clinical assessment of CTPs. Pharmacology, safety pharmacology, CKs, migration and persistence, toxicology and GLP are addressed [30].

Primary PD testing should be performed in appropriate disease models for the target clinical indication. In vitro studies of cell characteristics and functions are recommended if appropriate disease models are not available. The lowest or optimal effective dosage should also be explored. Secondary PD testing to assess unexpected pharmacological effects due to cell BD in non-targeted organs or the secretion of non-targeted bioactive factors is also addressed in the guidelines. Whether the CTP will be considered for safety pharmacology studies is determined on a case-by-case basis with the authority.

The guidelines highlight that PK absorption, distribution, metabolism and excretion studies for small-molecule drugs may not be suitable for CTPs. In this case, the investigator is recommended to assess the in vivo characteristics of the CTPs in terms of BD, viability, persistence and trafficking. Small animal models are considered appropriate for this purpose.

The requirements for toxicology studies of CTPs are also determined on a case-by-case basis, taking into consideration the manufacturing and characteristics of the CTPs, combination therapy, immunogenicity and immunotoxicity. In general, toxicology studies include general toxicity and local tolerance tests, and the dosing regimen should reflect the design of the clinical application. The suitability of the animal model for toxicology tests should also be considered. A single-dose toxicology study is generally sufficient; however, repeated-dose toxicology studies should be performed if the clinical application requires repeated doses. If required, local tolerance of the CTPs can be investigated along with the aforementioned general toxicity studies.

Tumorigenicity studies should be considered for stem cell products. Cells at the same passage (or beyond) as the defined CTP should be used for tumorigenicity studies. If, after discussion with the health authorities, it is determined that there are considerable findings during BD studies of the CTPs, further attention should be directed to specific organs or tissues.

Genotoxicity studies are generally not required unless there are concerns regarding direct interactions between CTP-secreted bioactive factors and DNA. Moreover, requirements for reproductive studies of the CTP will be considered on a case-by-case basis. For allogeneic CTPs, immunogenicity and immunotoxicity studies should be performed. In addition to the aforementioned guidelines, safety pharmacology and toxicology studies should be performed in compliance with GLP.

\section{Regulation in Japan}

To ensure the quality and safety of pharmaceuticals and medical devices derived from the processing of human cells of different autologous and allogeneic origin, seven notifications were issued by the Ministry of Health, Labour and Welfare (MHLW) in 2008 and 2012 [31-42]. The necessary non-clinical assessments prior to conducting clinical trials are briefly summarized as follows.

Regarding pharmacology studies, the guidelines state that potency testing should be performed to demonstrate functional expression, sustainability of effects and/or anticipated clinical efficacy (proof-of-concept). If animal disease models of the target indication are available for use, they should be utilized for assessing potential therapeutic efficacy. Notably, pharmacology studies can be waived before starting clinical studies if a significant efficacy superiority compared with that of other therapies can be justified based on the scientific literature. As for CK and BD studies, in vivo studies should be conducted to predict the characteristics in humans in terms of the survival period of the administered cells, efficacy duration, cell localization throughout the body and ROA validity.
The basic approach to the non-clinical safety evaluation of human CTPs is outlined in seven notifications [31-42], which state that relevant animal tests and/or in vitro tests should be performed to address safety concerns of the CTPs to the extent that they are scientifically reasonable and technically possible. Additionally, to clarify the safety assessment of CTPs, detailed technical guidelines for non-clinical safety evaluation, including general toxicity and tumorigenicity, were issued in 2016 [43]. The MHLW requires flexibly and rationally designed studies that consider the product characteristics on a caseby-case basis. Animal studies have scientific limitations in evaluating products of human origin and do not always yield meaningful results. In addition, manufacturing processes and cell sources of each product (e.g., embryonic stem cells, iPSCs, somatic stem cells and somatic cells) vary widely depending on product type. Generally, conducting quantitative risk assessment for CTPs is difficult; therefore, it is important to identify the hazards of the product as a potential safety concern, which can possibly be observed in vivo. Tumorigenic potential of the CTPs should also be assessed by considering the cell types and characteristics. For CTPs derived from pluripotent stem cells, their potential risk for malignant transformation or teratoma formation should be evaluated in tumorigenicity studies. Assessment is required for determining tumorigenicity of the raw material in CTPs as part of the quality characteristics and quality control tests, contamination of undifferentiated cells in the intermediate or final product and tumorigenicity potential of the final product at the human engraftment site. By contrast, according to the guidelines for tumorigenicity published in 2019 [44], in vivo tumorigenicity studies are not always necessary for CTPs derived from bone marrow-derived MSCs or somatic cells because the risk of tumorigenicity is confirmed to be low, especially for CTPs derived from somatic cells.

\section{Questionnaire Answers Regarding Virtual MSC-Derived CTPs}

The questionnaire was filled in according to local regulatory guidelines and development experiences. A high-level summary of answers regarding virtual MSC-derived CTPs is listed in Table 3, and details are included in supplementary Table 1 . The authors further investigated where answers were inconsistent to understand the different rationales of the respondents regarding non-clinical assessment for entering clinical trials. For virtual iPSC-derived CTPs, there were plenty of unclarified answers due to lack of regulatory guidelines and less experience, except for the respondents in Japan (data not shown). Therefore, the difference between MSCs and iPSCs is mainly focused on Japan.

\section{Points to Consider Before Clinical Application: Insights From the Questionnaire}

While investigating what is necessary and how assessments are done (e.g., to conduct the non-clinical studies), the authors were able to identify the differences that stemmed from different viewpoints across Asia. The authors were able to pinpoint different rationales regarding aspects of pharmacology, CK and BD studies, general toxicology and tumorigenicity (Figure 3), which will be described in the following sections. Moreover, considerations regarding virtual iPSCderived CTPs are suggested where relevant.

\section{Pharmacology}

In the real-world evaluation of CTPs, the required test items for non-clinical pharmacology studies differ depending on the product profile and clinical indication. In this case study, the authors conducted a survey of virtual CTPs with a well-defined product profile to avoid the complexities arising as a result of profile-dependent variabilities, which would hinder interpretation of the questionnaire. 
Table 3

High-level summary extracted from questionnaire answers by each respondent regarding virtual MSC-derived CTPs.

\begin{tabular}{|c|c|}
\hline Question & Main findings \\
\hline $\begin{array}{l}\text { Q: Are non-clinical evaluations (pharmacology, CK and BD studies and safety) } \\
\text { needed prior to starting clinical trials in your country/region? }\end{array}$ & - All respondents answered yes. \\
\hline Q: Please describe the type of studies (e.g., in vitro, in vivo, ex vivo) needed. & $\begin{array}{l}\text { - All respondents had similar answers except with regard to the tumorigenicity } \\
\text { study, for which Japan did not use an in vivo study. }\end{array}$ \\
\hline Q: Should the studies be conducted according to GLP? & $\begin{array}{l}\text { - Compliance with GLP is not required to conduct pharmacology and CK and BD } \\
\text { studies for CTPs but is required for safety assessments in most countries/ } \\
\text { regions. }\end{array}$ \\
\hline Q: How many animal species are required per study item? & $\begin{array}{l}\text { - One species of rodents (mice or rats) is accepted in most cases, but large animals } \\
\text { are also considered for use as a second animal species in China. } \\
\text { - The use of non-rodents (mainly rabbits) is also required for safety assessment in } \\
\text { India. }\end{array}$ \\
\hline Q: Which animal species and models are required for each study type? & $\begin{array}{l}\text { - Disease model is preferred to conduct pharmacology study. } \\
\text { - For safety studies, immunodeficient mice or rats are used in Korea and Japan. By } \\
\text { contrast, normal animals are used in India, China and Taiwan. }\end{array}$ \\
\hline $\begin{array}{l}\text { Q: What is the dosage to be tested and how many dose groups should be set per } \\
\text { study? }\end{array}$ & $\begin{array}{l}\text { - All respondents, with the exception of those in Japan, considered it necessary to } \\
\text { set two or three dose groups, with the highest dose at the MTD or MFD, for } \\
\text { pharmacology and safety assessment. }\end{array}$ \\
\hline $\begin{array}{l}\text { Q: Please describe the administration route, dosing regimen and items to be } \\
\text { assessed in non-clinical studies. }\end{array}$ & $\begin{array}{l}\text { - ROA corresponding to the clinical plan was recommended by all respondents. In } \\
\text { addition, systemic administration (intravenous and intramuscular) in India and } \\
\text { intravenous administration in China are required for safety assessment even if } \\
\text { ROA is intra-articular. } \\
\text { - Respondents in India, Korea and Taiwan stated that a dosing regimen similar to } \\
\text { clinical application is highly preferable. } \\
\text { - Combined study in pharmacology and safety assessment is highly recom- } \\
\text { mended among the respondents in China and Taiwan. }\end{array}$ \\
\hline Q: How long must the observational period be per study? & $\begin{array}{l}\text { - The respondents in India recommended performing a single-dose acute toxicity } \\
\text { study and repeat toxicity study for a } 2 \text {-week observation period after the last dose. } \\
\text { - The respondents in India and Korea set the observation period for chronic safety } \\
\text { assessment at } 13 \text { weeks after the last dose according to their guidelines. } \\
\text { - The respondents in India, China, Korea and Taiwan stated that the duration of } \\
\text { treatment in in vivo tumorigenicity study is basically around } 6 \text { months. }\end{array}$ \\
\hline
\end{tabular}

The answers prepared by each respondent generally agreed with regard to most parts of the questionnaire. Regarding the quality assurance of non-clinical pharmacology studies, all respondents answered that compliance with GLP is not required, but there was a consensus regarding adequate quality assurance. Regarding the selection of animal species and ROA for in vivo studies, there were some differences; some respondents required specific items to collate robust data in addition to those commonly required by all respondents. These additional items are not mandatory; however, they are proposed based on the idea that it is necessary to clarify the product profiles. Overall, the rationale in selecting pharmacology test items did not appear to differ among the respondents.

Despite the small differences in test items, the authors found a clear difference in setting dosing groups and dosages for animal studies. To clarify the different objectives of conducting a non-clinical pharmacology test, which could have caused the aforementioned differences, the authors conducted an additional questionnaire (see supplementary Table 2 ) in which the respondents were asked whether four objectives are mandatory for the IND-enabling pharmacology study of CTPs. Although all respondents recognized the necessity of non-clinical pharmacology tests, some differences became apparent. The respondents in Taiwan, Korea and Japan viewed it as mandatory to support the MOA of the product with an in vitro study, whereas the respondents in India and China did not. There were no differences regarding the objectives required by in vivo studies to support product efficacy in the clinical setting; however, other objectives, including the endpoints of non-clinical studies, differed. The respondents in India and Korea tended to have similar views of both non-clinical studies for CTPs and those for small molecules or biologics; therefore, defining onset time and duration, dose-response relationship and optimal dose is necessary. However, the respondents in Japan, China and Taiwan believed there is room to simplify the endpoints-in other words, showing the dose-response curve or defining the optimal dose in an in vivo study, for example, is not mandatory if the obtained non-clinical data appropriately describe the desired characteristics of the product and support initiating clinical trials. These differences are intriguing because they may reflect the different rationales of each local regulatory agency and not only those of the industry.

The authors conducted another survey to clarify how each respondent considers the extrapolation of cell doses from animals to
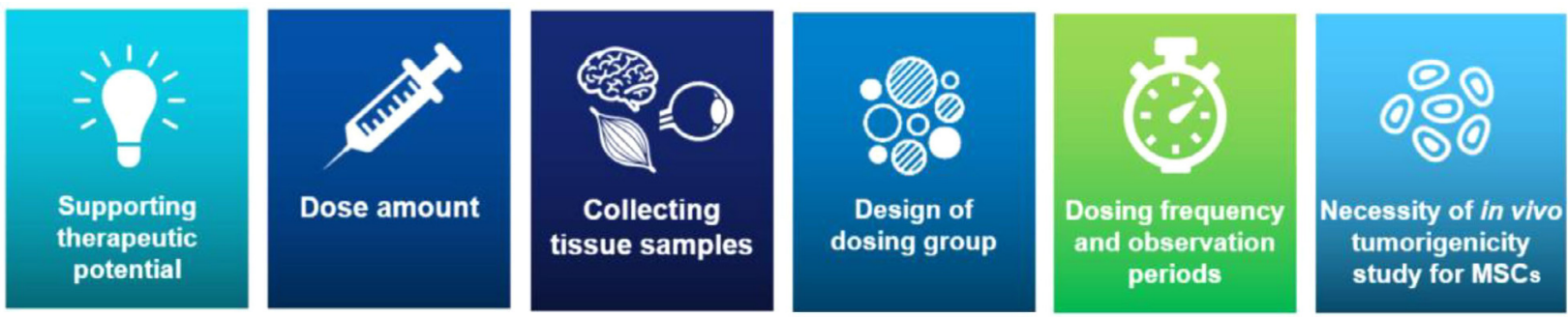

Figure 3. The main points where there were differences in perspective among the five Asian respondents. (Color version of figure is available online). 
humans. The authors asked the respondents whether they translate the optimal animal dose to humans or instead follow or refer to previous clinical studies of other products with a similar profile. All respondents recognized the difficulty in estimating the clinical dose from the optimal animal dose-specifically for cellular medicine-and preferred to refer to the clinical dose used in previous clinical trials of similar products. Nevertheless, at the same time, the respondents in India, Taiwan and Korea indicated that it is possible to set the clinical dose based on the optimal animal dose. Indeed, in Korea, thorough assessment of cell dose response and CKs is needed to support the cell dose for clinical trials. The respondent in India pointed out the importance of conducting dose-finding studies in humans and defining the dose response in animals.

For conventional medicine involving small molecules and biologics, clinical dose estimation using the PK/PD theory is applicable; however, its application in CTPs is associated with several problems. First, it is very difficult to evaluate CKs because of several methodological and technical limitations, such as species differences (including immune rejection), product-dependent BD and detection of surviving cells. Furthermore, for PD, it is difficult to clearly define the mechanisms underlying therapeutic effects because of the complex nature of cellular medicine. For example, in MSC-based medicine, including this virtual MSC-derived CTP in which the MOA is defined as paracrine, several secreted therapeutic molecules can be postulated as effector molecules; thus, estimation of their respective contribution and interaction is a big challenge even if their concentrations can be determined. Nevertheless, the evolution of CK technology, combined with an accumulation of clinical data regarding the therapeutic functions of cells, will lead to the development of PK/PD modeling for cellular medicine in the future.

\section{$C K$ and $B D$ studies}

CK and BD studies are essential for understanding the efficacy and toxicity profiles of CTPs. Based on the properties of the virtual CTPs and future clinical applications, the five respondents established their own non-clinical BD study settings. Regarding the common views, there were four main points: (i) compliance with GLP regulations is not required to conduct BD studies for CTPs; (ii) there was common preference for immunodeficient (immunosuppressant or immunocompromised) animals, as human-derived cells may induce an immune response in the models; (iii) ROA of the non-clinical study should correspond to the clinical plan (if intra-articular administration is assumed for the authors' virtual MSC-derived CTP in the clinic, then the non-clinical ROA should also be intra-articular; other ROAs, such as intravenous ROAs, are not considered necessary unless there is a particular concern with the CTP); and (iv) there were no differences in non-clinical BD study settings between MSC- and iPSC-derived CTPs. Although this is beyond the authors' focus at this time, a potential difference between the CTP types may be a longer monitoring period requested by the regulatory agencies for iPSC-derived CTPs, especially when virus integration is used.

In addition to the common viewpoints, the authors also identified several differences in BD study settings among the five respondents. One of the differences came from the question of how many animal species are required for BD studies. Most answers stated only one animal species, with immunodeficient mice or rats specified; however, the respondents in China included NHPs in their response. The use of large animals remains controversial. The advantage of employing NHPs for BD studies is the physiological similarity to humans, which provides more reliable data compared with the data achieved with small animals regarding the distribution and duration of CTPs. By contrast, prolonged observation times are difficult because immune rejection facilitates clearance of CTPs from the body. From a regulatory point of view, Food and Drug Administration and International Society for Stem Cell Research guidelines recommend the use of large animals, but European Medicines Agency guidelines emphasize the advantage of small animals while also stating the importance of similar evaluations in large animals for BD studies [45-48].

Another difference involves the choice of tissues to be investigated for BD studies of CTPs. Three out of five respondents have no specific guidelines for tissue selection; thus, tissues are to be selected while taking the characteristics of the CTPs into consideration. Furthermore, there are domestic guidelines in China and Korea regarding the specific tissues to be monitored. In China, blood, heart, lung, liver, kidney, brain, spleen and bone marrow samples as well as tissues at the local injection site should be monitored. The site of administration and target tissue, including brain, lung, liver, heart, spleen, testis/ovary, kidney, pancreas, bone marrow, blood and lymph nodes, are specified in the Ministry of Food and Drug Safety guidelines of Korea. To the authors' knowledge, there are currently no other guidelines on the selection of specific tissues for BD studies of CTPs. It is expected that technical advances in bioimaging will help select the optimal tissues for performing quantitative polymerase chain reaction with increased sensitivity.

With regard to the question of what the appropriate dosage for non-clinical BD studies is, the authors obtained two answers: MFD or human clinical dose. One of the reasons for supporting MFD, as stated by the respondents in Japan, is that the dose should be consistent with the toxicity studies. The respondents in Korea selected the human clinical dose when the extrapolated dose from the animal model is below the MFD. In addition, the dosage should generally be dependent on the purpose of the BD study (e.g., to understand the pharmacological or toxicological profiles of the CTPs).

Regarding whether single or multiple doses are better for non-clinical $\mathrm{BD}$ studies, the five respondents determined the optimal dose frequency based on the assumption that the clinical trial would be set at multiple doses for the virtual MSC-derived CTP. The Ministry of Food and Drug Safety guidelines in Korea recommend that the non-clinical dosage should follow the clinical course, and thus multiple doses were selected in this case. Some of the other respondents agreed with this idea. However, a single dose was preferred when the administration interval was long enough and cells were cleared from the body, as the results can allow for a simulation of CKs after multiple doses. The respondents in Taiwan recommended both multiple and single doses to assess cellular properties because data from a single-dose study can first reveal the cell characteristics without factors such as accumulation or immunological memory responses even though the clinical setting would involve multiple doses. A reasonable comment was made by the respondents in Japan, who stated that the clinical dose should originally be planned based on the cellular characteristics revealed in non-clinical studies and that it does not make much sense that the clinical setting is considered first.

As a result of the survey, the authors were able to identify the specific points to be considered in non-clinical BD studies for IND submission. All answers by the five respondents appeared reasonable, and there may even be more answers, as this is an immature field. It is assumed that the study settings are determined on a case-by-case basis according to the properties of the CTP candidates and that decisions are implemented after discussion with the regulatory agencies. Although it may be technically challenging to perform clinical BD studies, non-clinical findings are very useful for evaluating the potential distribution and lifetime of CTPs in humans. It is thus expected that non-clinical BD results will help develop an appropriate clinical dose regimen or strategy to avoid critical toxicity in humans.

\section{General toxicology}

The design of general toxicity studies reflects the requirements of the local regulatory agency guidelines. Generally, uniform standards similar to those for chemically synthesized drugs are required in India, China, Korea and Taiwan, whereas the Japanese guidelines allow for a flexible study design based on scientific rationale in terms 
Table 4

General toxicity study designed by each respondent with regard to the virtual MSC-derived CTPs.

\begin{tabular}{|c|c|c|}
\hline Respondent & General toxicity study & Immunogenicity test \\
\hline India & $\begin{array}{l}\text { 1. A 2-week repeated daily IV administration study in normal rats. } \\
\text { - Repeated administration every day over } 2 \text { weeks via IV is required followed by additional observation period for } 2 \text { weeks. } \\
\text { - It may follow the clinical trial protocol (i.e., repeated administration for six times in this case). } \\
\text { - One dose group is acceptable. } \\
\text { 2. Single acute IA and IV/IM administration study in both normal rats and rabbits. } \\
\text { - IA (two animals: rats and rabbits) and both IV (two animals: rats and rabbits) and IM (two animals: rats and rabbits) studies are } \\
\text { required followed by 2-week observation period after a single dose. } \\
\text { - Multiple dose groups are set. } \\
\text { - IA is mandatory, but if pharmacology study is conducted via IA route, it could be combined. } \\
\text { 3. Single subchronic IV/IM administration study in normal rats and rabbits. } \\
\text { - Both IV (two animals: rats and rabbits) and IM (two animals: rats and rabbits) studies are required followed by 13-week observa- } \\
\text { tion period after a single dose. } \\
\text { - Multiple dose groups are set. } \\
\text { - IA route is not mandatory. }\end{array}$ & Yes \\
\hline China & $\begin{array}{l}\text { 1. Repeated IA and IV administration study in relevant normal small animal model (e.g., rats) and large animal model (e.g., monkeys). } \\
\text { - IA (both small and large animals) and IV (both small and large animals) studies, with administration repeated six times to mimic } \\
\text { clinical setting, are required followed by } 12 \text {-week observation period after the last dose. } \\
\text { - Multiple dose groups are set. } \\
\text { - Monkey model is optional but better to use. } \\
\text { 2. Single IA and IV administration study in relevant normal small animal model (e.g., rats) and large animal model (e.g., monkeys). } \\
\text { - IA (both small and large animals) and IV (both small and large animals) studies are required followed by 4-week observation } \\
\text { period after a single dose. } \\
\text { - Multiple dose groups are set. } \\
\text { - Monkey model is optional but better to use. }\end{array}$ & Yes \\
\hline Korea & $\begin{array}{l}\text { 1. Repeated IA administration study in immunodeficient rats. } \\
\text { - Biweekly administration for } 13 \text {-week period is considered followed by } 13 \text {-week observation period after the last dose. } \\
\text { - Multiple dose groups considering MFD are required to understand dose-response relationship. } \\
\text { - Repeated administration is required according to the clinical protocol regardless of BD data. } \\
\text { - Observation period might be shortened based on BD study. }\end{array}$ & Yes \\
\hline Taiwan & $\begin{array}{l}\text { 1. Repeated IA administration study in normal rats. } \\
\text { - Repeated administration six times with 2-week intervals is required followed by } 1 \text {-month observation period after the last dose. } \\
\text { - Multiple dose groups are set. }\end{array}$ & Yes \\
\hline Japan & $\begin{array}{l}\text { 1. Repeated IA administration study in immunodeficient rats. } \\
\text { - Repeated administration two times once a month is considered followed by } 2 \text { - or } 4 \text {-week observation period after the last dose. } \\
\text { - Single administration is able to be adapted to assess a reasonable hazard risk if no accumulation in the body is confirmed in CK and } \\
\text { BD study before the general toxicity study. }\end{array}$ & No \\
\hline
\end{tabular}

IA, intra-articular; IM, intramuscular; IV, intravenous.

of product characteristics or MOA. Survey results of general toxicity are summarized in Table 4.

Regarding the dose selection, all respondents, with the exception of those in Japan, considered it necessary to set two or three dose groups, with the highest dose at the MTD or MFD, to identify the MTD of the test substance and determine the dose-dependent toxicity. Even if the product does not show bioaccumulation in vivo, the guidelines require a repeated-dose toxicity study for products that are intended to be used with repeated administration in the clinic. However, the respondents in Japan considered that only one dose group at the MTD or MFD is sufficient from the point of hazard risk assessment. The Japanese guidance is that quantitative risk assessment is difficult to apply for CTPs because human-derived CTPs possibly induce a heterologous immune response in animals and species differences in the biological activity of each bioactive substance (e.g., cytokines) can arise. Therefore, the respondents in Japan concluded that at least two dose groups (i.e., a control group and a treatment group at the MTD or MFD) are sufficient for confirming the product hazard risk.

Regarding the number of doses, all respondents, with the exception of those in Japan, stated that they would follow the regimen planned for clinical use and administer the same six repeated doses in a repeated-dose animal study. This is because their guidelines require the number of doses to be consistent with those for clinical use. By contrast, the respondents in Japan set two doses for confirming bioaccumulation in vivo; the rationale is that even a single dose would be acceptable if other studies, such as BD and preliminary toxicology studies, have shown no accumulation in vivo, as specified in the Japanese guidance. Thus, a safety assessment should be conducted in which as many CTPs as possible are administered while considering that the toxicity will not be exacerbated unless the CTPs show accumulation in vivo. The respondents in Japan assumed that this virtual product would show no accumulation in vivo and therefore judged that the two doses were enough for assessment.

Regarding dosing, frequency and study period, the respondents in Japan answered that administration frequency should be monthly, similar to the clinical dosing frequency, and set an observation period of 14 days to 1 month after the second administration, with a total study period of $1.5-2$ months. The Japanese guidance mentions that a minimum observation period of 14 days is sufficient to evaluate systemic toxicity of CTPs because of the limitations of safety evaluation in which a xenogeneic immune response occurs. However, if there is a specific safety concern associated with the MOA of the product, an appropriate observation period based on the results of a pharmacology assessment should be set. The respondents in China and Taiwan answered that chronic toxicity should be assessed alongside a pharmacology study and that the study period would be determined in consideration of safety concerns arising from the MOA and duration of the product remaining in the body. The respondents in both Korea and India determined a 13-week observation period, which is the standard observation period stated in their guidelines; this period can be shortened in Korea depending on the BD study results.

In addition to repeated-dose toxicity studies, the guidelines in India require a single-dose acute toxicity study to be performed in normal rats and rabbits, respectively, with an observation period of 14 days. For Taiwan, a study employing normal rats is mandatory to confirm the response of normal animals to human-derived CTPs for acute toxicity assessment. 
Tumorigenicity

\section{Tumorigenicity assessment of MSC-derived CTPS}

For tumorigenicity risk assessment of MSC-derived CTPs, a critical difference appeared between the respondents in Japan and the others regarding the necessity of in vivo studies. Although all respondents, with the exception of those in Japan, mandated both in vitro and in vitro studies, the respondents in Japan set only an in vitro study for tumorigenicity assessment. In previous clinical experiences with MSC-derived CTPs in Japan, tumor formation has not been reported, and it is believed that the proliferation activity of MSCs is lower than that seen with iPSC-derived products. Therefore, the respondents in Japan consider in vitro studies adequate for confirming the absence of tumorigenic cell contamination of the final products and for determining cell growth characteristics in tumorigenicity assessment. For the other respondents, the tumorigenicity of MSC-derived CTPs is recognized to be low, but the guidelines, including draft guidelines, require in vivo tumorigenicity studies.

For in vitro studies, karyotyping and/or soft agar colony formation studies were required. For all respondents, with the exception of those in China, non-GLP grades are acceptable for the studies. In addition, for in vivo studies, all respondents, with the exception of those in India, required that the studies be conducted according to GLP standards. Regarding animal species, immunocompromised mice or rats are required, and for Korea, the use of an animal disease model is also recommended if applicable. Although selecting the dosage and determining the highest dose, such as MFD, MTD or $\geq 10$ times the clinical dose, differed among the respondents, setting multiple dose groups was a common response. In terms of detecting a tumorigenic hazard, assessment of only one dose group administered the MFD or MTD is sufficient in Japan, whereas evaluation of multiple dose groups is required in the other countries/regions. For the respondents who required in vivo studies, a positive control dose group is necessary to confirm establishment of the study condition. Details regarding the positive control are discussed in the following section. The respondents in Korea, Taiwan and India described that the observation period was set at 6 months, whereas the respondents in China answered that it was dependent on the characteristics of MSC-derived CTPs and clinical indications. The respondents in Korea also indicated that it is possible to shorten the observation period in cases with a shorter residual period of MSCderived CTPs in the body. The respondents in Taiwan considered 6 months a sufficient evaluation period, as it covers one-fourth to onehalf of the lifetime of the model animals and $>6$ months would increase the risk of spontaneous tumor formation. The guidelines in India specify an observation period of 6 months.

\section{Tumorigenicity assessment of iPSC-derived CTPS}

When introduced in their pluripotent state, iPSCs are intrinsically tumorigenic and form teratomas in immunodeficient animals. Therefore, contamination with undifferentiated iPSCs is considered a major tumorigenicity risk of iPSC-derived CTPs. Indeed, most of the respondents (India, Korea, Taiwan and Japan) recognized that in vitro assessment of residual undifferentiated iPSCs was needed for the virtual iPSCderived CTPs. However, this questionnaire did not include further details regarding which types of assays are generally considered for residual undifferentiated iPSC assessment; for instance, polymerase chain reaction-based and/or culture-based assays are generally conducted in Japan [40]. Regarding other in vitro tumorigenicity assays, the respondents in India, Taiwan and Japan selected karyotyping, as virtual iPSC-derived CTPs are blood cell-derived floating cells; however, soft agar colony formation assay, a common tumorigenicity assay required by almost all respondents to detect anchorage-independent growth for MSC-derived CTPs, was not chosen for this setting. By contrast, the respondents in Korea and China selected both karyotyping and soft agar colony formation assay for in vitro tumorigenicity assessment.
A Japanese guidance issued by the MHLW in 2016 [43] indicates that the tumorigenicity concern regarding iPSC-derived CTPs is higher than that for somatic stem cells or somatic cell-derived CTPs; this is because of the genomic instability risk resulting from the high extent of manipulation during the reprogramming process and the teratoma formation risk from residual undifferentiated iPSCs, as previously described. Therefore, in contrast to MSC-derived CTPs, all respondents, including those in Japan, considered in vivo tumorigenicity studies necessary. The duration of the observation period proposed by the respondents in India, China, Korea and Taiwan was 6 months, which was the same as that proposed for virtual MSC-derived CTPs; however, the respondents in Japan took into consideration the high tumorigenicity risk of iPSCderived CTPs and set a longer observation period that covered the life span of the animal and/or the time needed until the administered cells disappeared from the body. In terms of the dose, multiple doses are required by all respondents, other than those in Japan, even though the MFD/MTD is considered less than the absolute cell number of clinical doses $\left(1 \times 10^{9}\right.$ cells/body). This is because the equivalent human dose can be converted based on the body weight or tissue volume/area of target tissues for CTPs in India, Korea and Taiwan. In addition, one dose level of MFD/MTD is considered sufficient in Japan because non-clinical studies of CTPs are generally conducted for hazard assessment. Another difference between the respondents in Japan and the others was the necessity of a positive control group in in vivo tumorigenicity studies. As noted earlier for MSC-derived CTPs, all respondents, with the exception of those in Japan, required a positive control group for in vivo tumorigenicity studies to confirm validity of the study design. However, the respondents in Japan considered in vivo tumorigenicity studies using immunodeficient animals an established study design, and thus a positive control group is not mandatory. Another reason the respondents in Japan indicated for not requiring a positive control group is the difficulty in choosing appropriate positive control cells based on the CTPs. Ideally, positive control cells should reflect the characteristics of the CTPs as much as possible. Furthermore, control cells are required to be readily available, well characterized and of consistent quality. The respondents in Japan recommend tumor cell lines with similar origins or product characteristics as positive controls. By contrast, the respondents in India, China and Taiwan accept any type of cancer cells that are confirmed to form tumors with a high incidence under the conditions of the study.

\section{Immunogenicity}

In Korea, immunogenicity concerns should be addressed for allogeneic MSC-based therapies via in vitro and in vivo experimental evidence. Characterization of immune properties by HLA typing and allogeneic mixed lymphocyte reaction is recommended for in vitro studies. Animal disease models reflecting the clinical settings are recommended for in vivo studies, which can be combined with general toxicity studies. If available, animals with reduced xenogeneic immune reactions or analogous animal cells with characteristics similar to the target human cells should be considered. In China, immunogenicity should be evaluated for allogeneic CTPs through in vitro and in vivo animal studies, which includes assessing the effects on different immune cell subtypes and related cytokines. For embryonic stem cells and iPSC-derived CTPs, donor HLA antigens are reexpressed after differentiation in vitro, and thus the risk of immune rejection needs to be carefully evaluated.

\section{Discussion}

The importance of gathering and sharing data among countries/ regions has been emphasized multiple times and identified as a requirement for the successful development of safe and effective cell-based therapies. Generally, non-clinical assessment is designed on a case-by-case basis according to the nature of the CTPs. Here the authors used two virtual CTPs (MSC- and iPSC-derived) and were 


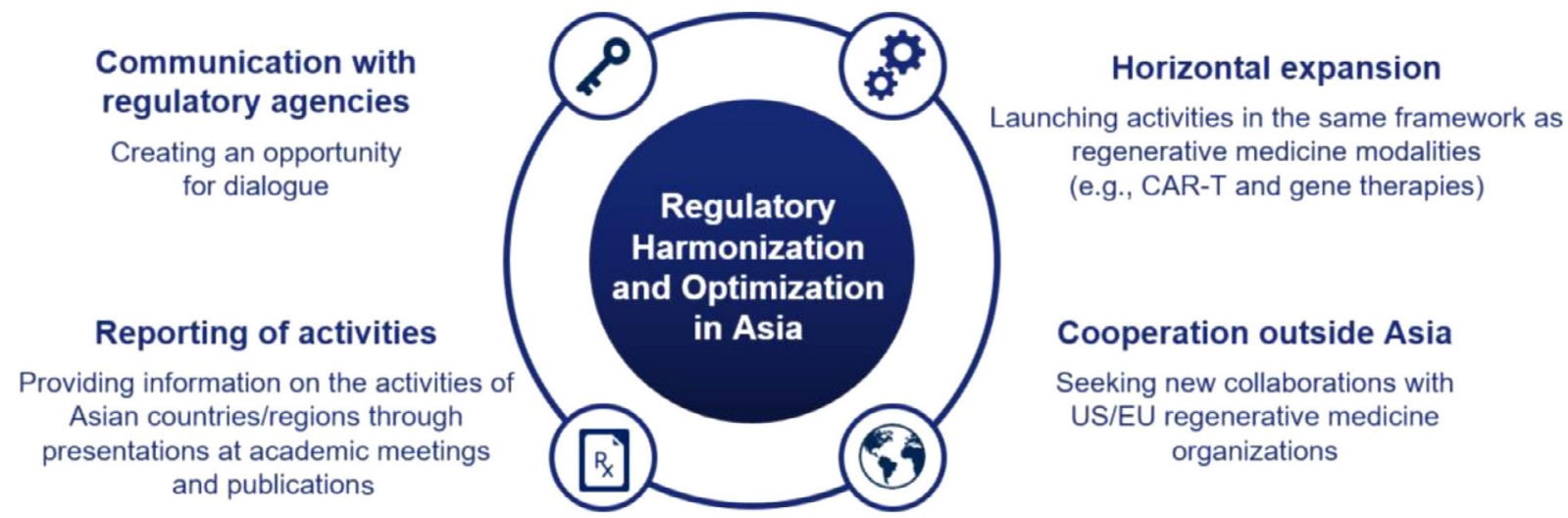

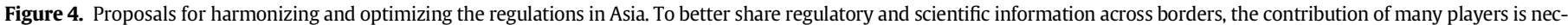

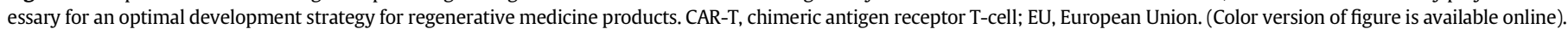

able to identify the different perspectives of five Asian countries/ regions (India, China, Korea, Taiwan and Japan) in terms of setting the non-clinical assessments required before the products can enter clinical trials. Through this survey, the regulatory landscape and varying levels of development experience of each participating respondent were found to affect the non-clinical data package and study design for ensuring safety and efficacy in humans. Thus, the authors' findings help us understand the specific case-by-case strategies adopted by the local regulatory agencies and can help optimize regulatory science in Asia by sharing information regarding the non-clinical evaluation necessary for human CTPs.

However, the characteristics of the virtual CTPs in this survey are only limited examples. The expertise of APACRM WG1 is also limited to non-clinical assessments. In the future, considering virtual products of other regenerative medicine modalities (e.g., chimeric antigen receptor T-cell and gene therapy) as well as multiple aspects (e.g., quality control, clinical trial assessment or post-marketing strategies) can be valuable. The authors also allow the open access to APACRM information for Malaysia, Thailand and other East Asian countries so that they have their interest to this activity. The authors are looking forward to the future participation of these countries. Ideally, collaboration with the industries of other countries (e.g., the US and European Union), and not just the ones included here, would improve our understanding of what is needed for non-clinical assessment beyond Asia.

Sharing knowledge and collating data based on both the industry and the regulatory authority will enable us to rapidly deliver CTPs to more patients and achieve market maximization of CTPs in Asia. Therefore, the authors suggest creating an open platform (e.g., workshops and annual conferences) for discussing regulatory science matters with the relevant regulatory agencies. Continuous discussions with health authority regulators and sponsors in Asia are indispensable for facilitating advancement of CTP development. To this end, the authors hope that the environment of harmonization is cultivated among regulatory agencies via the sharing of experiences with products under development and the exchange of opinions regarding the regulatory guidelines of each country/region. Moreover, a more proactive contribution to regulatory science (e.g., the necessity of new guidelines) regarding regenerative medicine products will help regulatory harmonization and optimization in Asia. The authors' proposals are conceptually described in Figure 4.

\section{Funding}

This work was supported by the Forum for Innovative Regenerative Medicine. The Forum for Innovative Regenerative Medicine had no involvement in the study design; collection, analysis or interpretation of data; or writing of the article.

\section{Author Contributions}

Conception and design of the study: TY, YF, HK, YM, KB, YK, MK, KO, TW and YT. Acquisition of data; Analysis and interpretation of data; and Drafting or revising the manuscript: TY, BHC, PKG, CYH YPT, LMW, YF, HK, YM, KB, YK, MK, KO, TW, YB, FCC, DSH, JYL, GL, YL, JO and YT. All authors have approved the final article.

\section{Declaration of Competing Interest}

All authors, with the exception of BHC, are employees of companies developing cellular therapies, providing testing or enabling technologies for the manufacture of cellular therapies.

\section{Acknowledgments}

The authors are grateful to Dr Masayuki Nomura (chair of the international affairs committee at the Forum for Innovative Regenerative Medicine), Ms Ying Gai (China Medicinal Biotech Association) and Ms Suzan Lin (Biotechnology and Pharmaceutical Industries Promotion Office) for supporting WG1. The authors would like to thank Editage (www.editage.com) for English language editing.

\section{Supplementary materials}

Supplementary material associated with this article can be found in the online version at doi:10.1016/j.jcyt.2021.04.007.

\section{References}

[1] Chen C, Dubin R, Kim MC. Emerging trends and new developments in regenerative medicine: a scientometric update (2000-2014). Expert Opin Biol Ther 2014;14(9):1295-317.

[2] Fitzsimmons REB, Mazurek MS, Soos A, Simmons CA. Mesenchymal Stromal/Stem Cells in Regenerative Medicine and Tissue Engineering. Stem Cells Int 2018;2018:8031718.

[3] Hanna E, Remuzat C, Auquier P, Toumi M. Advanced therapy medicinal products: current and future perspectives. J Mark Access Health Policy 2016:25:4.

[4] Cuende N, Rasko JEJ, Koh MBC, Dominici M, Ikonomou L. Cell, tissue and gene products with marketing authorization in 2018 worldwide. Cytotherapy 2018;20 (11):1401-13.

[5] Petricciani J, Hayakawa T, Stacey G, Trouvin JH, Knezevic I. Scientific considerations for the regulatory evaluation of cell therapy products. Biologicals 2017;50:20-6

[6] Iglesias-Lopez C, Agusti A, Obach M, Vallano A. Regulatory Framework for Advanced Therapy Medicinal Products in Europe and United States. Front Pharmacol 2019;10:921.

[7] Han F, Wang J, Ding L, Hu Y, Li W, Yuan Z, et al. Tissue Engineering and Regenerative Medicine: Achievements, Future, and Sustainability in Asia. Front Bioeng Biotechnol 2020;8:83.

[8] Chen Y, Chen Y, Tsai P, Chen C, Shaw S, Hsuan Y. Mesenchymal stem cell: considerations for manufacturing and clinical trials on cell therapy product. Int J Stem Cell Res Ther 2016:3(1):029. 
[9] Doss MX, Sachinidis A. Current Challenges of iPSC-Based Disease Modeling and Therapeutic Implications. Cells 2019;8(5):403.

[10] Azuma K, Yamanaka S. Recent policies that support clinical application of induced pluripotent stem cell-based regenerative therapies. Regen Ther 2016;4:36-47.

[11] Shi Y, Inoue H, Wu JC, Yamanaka S. Induced pluripotent stem cell technology: a decade of progress. Nat Rev Drug Discov 2017;16(2):115-30.

[12] Takahashi K, Tanabe K, Ohnuki M, Narita M, Ichisaka T, Tomoda K, et al. Induction of pluripotent stem cells from adult human fibroblasts by defined factors. Cell 2007; 131(5):861-72.

[13] de Hair MJH, Jacobs JWG, Schoneveld JLM, van Laar JM. Difficult-to-treat rheumatoid arthritis: an area of unmet clinical need. Rheumatology (Oxford) 2018;57 (7):1135-44

[14] Sardar S, Andersson A. Old and new therapeutics for Rheumatoid Arthritis: in vivo models and drug development. Immunopharmacol Immunotoxicol 2016;38 (1):2-13.

[15] Naji A, Eitoku M, Favier B, Deschaseaux F, Rouas-Freiss N, Suganuma N. Biologica functions of mesenchymal stem cells and clinical implications. Cell Mol Life Sci 2019;76(17):3323-48.

[16] Fan XL, Zhang Y, Li X, Fu QL. Mechanisms underlying the protective effects of mesenchymal stem cell-based therapy. Cell Mol Life Sci 2020;77(14):2771-94.

[17] Indian Council of Medical Research. National Guidelines of Stem Cell Research, http://dbtindia.gov.in/sites/default/files/National_Guidelines_StemCellResearch2017.pdf; 2017 [accessed November 23 2020] 2021.

[18] The Central Drugs Standard Control Organization. New Drug and Clinical Trial Rules, https://cdsco.gov.in/opencms/export/sites/CDSCO_WEB/Pdf-documents/ NewDrugs_CTRules_2019.pdf; 2019 [accessed November 23 2020].2021

[19] National Medical Products Administration. Guidance on CMC and preclinica study of stem cell, https://www.nmpa.gov.cn/xxgk/fgwj/gzwj/gzwjyp 20150731120001226.html; 2015 [accessed November 23 2020]. 2021

[20] National Medical Products Administration. Guidance on cell therapy products study and evaluation (Drafted), https://www.nmpa.gov.cn/directory/web/nmpa/ xxgk/ggtg/qtggtg/20171222145101557.html; 2017 [accessed November 23 2020]. 2021

21] International conference on harmonisation of technical requirements for registration of pharmaceuticals for human use. ICH guideline S6 (R1)-preclinical safety evaluation of biotechnology-derived pharmaceuticals, https://admin.ich.org/ sites/default/files/inline-files/S6_R1_Guideline.pdf; 2011 [accessed November 14 2020]. 2021

[22] Ministry of Food and Drug Safety. Guidance on Non-clinical Assessment of Gene Therapies, https://www.law.go.kr/법령/첨단재생의료및첨단바이오의약품안전및지 원 에관한법률; 2020 [accessed November 23 2020]. 2021

[23] Ministry of Food and Drug Safety. Guidance on Non-clinical Assessment of Gene Therapies, http://www.nifds.go.kr/brd/m_15/down.do?brd_id=167\&seq=11915\&data tp=A\&file_seq=1; 2017 [accessed November 23 2020]. 2021

[24] Ministry of Food and Drug Safety. B1-2014-3-020 Guideline in Quality, Non-clinical and Clinical Assessment of Stem Cell Therapy Product, http://www.nifds.go. $\mathrm{kr} / \mathrm{brd} / \mathrm{m} \_158 /$ down.do?brd_id=235\&seq=11723\&data_tp=A\&file_seq=1; 2014 [accessed November 23 2020]. 2021

[25] Ministry of Food and Drug Safety. B1-2014-3-005 Considerations in Immunotoxicity Assessment of Allogenic Stem Cell Therapy Product, https://www.nifds.go $\mathrm{kr} / \mathrm{brd} / \mathrm{m} \_158 /$ down.do?brd_id=235\&seq=11727\&data_tp=A\&file_seq=1; 2014 [accessed November 23 2020]. 2021

[26] Berglund AK, Fortier LA, Antczak DF, Schnabel LV. Immunoprivileged no more: measuring the immunogenicity of allogeneic adult mesenchymal stem cells. Stem Cell Res Ther 2017:8(1):288.

[27] Lohan P, Treacy O, Griffin MD, Ritter T, Ryan AE. Anti-Donor Immune Responses Elicited by Allogeneic Mesenchymal Stem Cells and Their Extracellular Vesicles: Are We Still Learning? Front Immunol 2017;8:1626.

[28] Ministry of Food and Drug Safety. B1-2014-3-006 Considerations in Tumorigenicity Assessment of Stem Cell Therapy Product, https://www.nifds.go.kr/brd/ m_158/down.do?brd_id=235\&seq=11721\&data_tp=A\&file_seq=1; 2014 [accessed November 23 2020]. 2021

[29] Ministry of Food and Drug Safety. Considerations in Biodistribution Assessment of Stem Cell Therapy Product, http://www.nifds.go.kr/brd/m_158/down.do? brd_id=235\&seq=11722\&data_tp=A\&file_seq=1; 2014 [accessed November 23 2020]. 2021

[30] Taiwan Food and Drug Administration. Guidance on Investigational Cell Therapy Products, https://www.fda.gov.tw/tc/includes/GetFile.ashx?id=f637241904805234740; 2020 [accessed November 27 2020]. 2021

[31] Pharmaceuticals and Medical Devices Agency. Guidelines on Ensuring the Quality and Safety of Pharmaceuticals and Medical Devices Derived from the Processing of Autologous Human Cell/Tissue, https://www.pmda.go.jp/files/000205396.pdf: 2008 [accessed October 27 2020]. 2021
[32] Pharmaceuticals and Medical Devices Agency. Guidelines on Ensuring the Quality and Safety of Pharmaceuticals and Medical Devices Derived from the Processing of Allogeneic Human Cell/Tissue, https://www.pmda.go.jp/files/000205398.pdf; 2008 [accessed October 27 2020]. 2021

[33] Pharmaceuticals and Medical Devices Agency. Guidelines on Ensuring the Quality and Safety of Pharmaceuticals and Medical Devices Derived from the Processing of Autologous Human Somatic Stem Cells, https://www.pmda.go.jp/files/ 000205400.pdf; 2012 [accessed October 27 2020]. 2021

[34] Pharmaceuticals and Medical Devices Agency. Guidelines on Ensuring the Quality and Safety of Pharmaceuticals and Medical Devices Derived from the Processing of Allogeneic Human Somatic Stem Cells, https://www.pmda.go.jp/files/ 000205401.pdf; 2012 [accessed October 27 2020]. 2021

[35] Pharmaceuticals and Medical Devices Agency. Guidelines on Ensuring the Quality and Safety of Pharmaceuticals and Medical Devices Derived from the Processing of Autologous Human Induced Pluripotent Stem (-like) Cells, https://www.pmda. go.jp/files/000205401.pdf; 2012 [accessed October 27 2020]. 2021

[36] Pharmaceuticals and Medical Devices Agency. Guidelines on Ensuring the Quality and Safety of Pharmaceuticals and Medical Devices Derived from the Processing of Allogeneic Human Induced Pluripotent Stem (-like) Cells, https://www.pmda. go.jp/files/000205403.pdf; 2012 [accessed October 27 2020]. 2021

[37] Pharmaceuticals and Medical Devices Agency. Guidelines on Ensuring the Quality and Safety of Pharmaceuticals and Medical Devices Derived from the Processing of Human Embryonic Stem Cells, https://www.pmda.go.jp/files/000205404.pdf; 2012 [accessed October 27 2020]. 2021

[38] Hayakawa T, Aoi T, Umezawa A, Ozawa K, Sato Y, Sawa Y, et al. A study on ensuring the quality and safety of pharmaceuticals and medical devices derived from the processing of autologous human somatic stem cells. Regen Ther 2015;2:57-69.

[39] Hayakawa T, Aoi T, Umezawa A, Ozawa K, Sato Y, Sawa Y, et al. A study on ensuring the quality and safety of pharmaceuticals and medical devices derived from the processing of allogeneic human somatic stem cells. Regen Ther 2015;2:70-80.

[40] Hayakawa T, Aoi T, Umezawa A, Ozawa K, Sato Y, Sawa Y, et al. A study on ensuring the quality and safety of pharmaceuticals and medical devices derived from processing of autologous human induced pluripotent stem(-like) cells. Regen Ther 2015;2:81-94.

[41] Hayakawa T, Aoi T, Umezawa A, Ozawa K, Sato Y, Sawa Y, et al. A study on ensuring the quality and safety of pharmaceuticals and medical devices derived from processing of allogeneic human induced pluripotent stem(-Like) cells. Regen Ther 2015;2:95-108.

[42] Hayakawa T, Aoi T, Umezawa A, Ozawa K, Sato Y, Sawa Y, et al. A study on ensuring the quality and safety of pharmaceuticals and medical devices derived from the processing of human embryonic stem cells. Regen Ther 2015;2:109-22.

[43] Pharmaceuticals and Medical Devices Agency. Technical Guidance on the implementation of the Quality, Nonclinical and Clinical Study of Regenerative Medicine, https:// www.pmda.go.jp/files/000212850.pdf; 2016 [accessed October 27 2020]. 2021

[44] Pharmaceuticals and Medical Devices Agency. Guidelines on the Detection of Undifferentiated Pluripotent Stem Cells and Transformed Cells, tumorigenicity tests and genetic stability evaluation on Human Cell Processed Products, https:// www.mhlw.go.jp/hourei/doc/tsuchi/T190705I0010.pdf; 2019 [accessed October 27 2020]. 2021

[45] US Food and Drug Administration. Guidance for Industry: Preclinical Assessment of Investigational Cellular and Gene Therapy Products, https://www.fda.gov/ media/87564/download; 2013 [accessed November 13 2020]. 2021

[46] International Society For Stem Cell Research (ISSCR). Guidelines for stem cell research and clinical translation, https://www.isscr.org/policy/guidelines-for-stemcell-research-and-clinical-translation; 2016 [accessed November 13 2020]. 2021

[47] European Medicines Agency. Guideline on human cell-based medicinal products, https://www.ema.europa.eu/en/documents/scientific-guideline/guidelinehuman-cell-based-medicinal-products_en.pdf; 2007 [accessed November 13 2020]. 2021

[48] European Medicines Agency. Reflection paper on stem cell-based medicinal products, https://www.ema.europa.eu/en/documents/scientific-guideline/reflectionpaper-stem-cell-based-medicinal-products_en.pdf; 2011 [accessed November 13 2020]. 2021

[49] The Central Drugs Standard Control Organization. SPS/2013-001 Guidance Document for Regulatory Approvals of Stem Cell and Cell Based Products (SCCPs), https://auroushealthcare.files.wordpress.com/2014/02/guidance-document-forstem-cells-and-cell-based-products_cdsco.pdf; 2013 [accessed November 23 2020]. 2021

[50] Center for Drug Evaluation. Draft technical guidelines for clinical trials of humanderived stem cells and cell therapy products, http://www.cde.org.cn/news.do? method=largeInfo\&id=6cf5b4fbef3256d9; 2020 [accessed November 23 2020]. 2021 